\title{
BINARY EVOLUTION IN THE UPPER HRD
}

\author{
J.P. De Greve and C. de Loore* \\ Astrophysical Institute VUB \\ Brussels, Belgium. \\ *Also University of Antwerp, RUCA.
}

\section{ABSTRACT .}

The importance of close binaries in the domain of luminous objects is examined. The group of unevolved massive stars of $0-$ and B-type, the groups of Wolf-Rayet binaries and X-ray binaries are discussed, and limits on the mass ratios, masses and periods are listed, as well as the average values. The connections between these various groups are investigated, and relations among subgroups are established.

\section{INTRODUCTION.}

An attempt is made to clarify the importance of close binaries in the field of luminous objects, importance which may be expressed either in view of the importance of the fraction of binaries among luminous objects, or with regard to the information they offer, or both.

Three large groups of massive close binaries are examined: the 0type binaries, the Wolf-Rayet binaries and the X-ray binaries.

For each of these groups, a selection of "well as defined" systems is made, and characteristic values, as well averages, and observed lower and upper boundery values for masses, mass ratios, luminosities and periods, are established.

Different theoretical possiblities for close binary evolution, with or without mass exchange, conservative or taking into account mass and angular momentum losses are discussed.

Models for massive binaries are analysed. The difficulties related to the determination of the ancestors for well known actual systeins are stressed, in view of the uncertainties concerning stellar wind mass losses during the main sequence stage, the fraction of mass and angular momentum losses during the Roche lobe overflow phase, and the stellar wind mass losses during the Wolf-Rayet phase.

Constraints on the mass ratio and periods for observed systems allow to determine a discriminating mass for systems evolving into $X$-ray sources. 
2. GROUPS OF MASSIVE BINARIES.

2.1. If the term 'massive binary' is used in a strict sense, three groups can be distinguished.

a) Unevolved massive binaries (i.e. no past or present interaction can be deduced from the observations).

Garmany, Conti and Massey (1980) list 40 0-type binaries, 29 of which are double-lined, while de Jager (1980) gives a list of 30 systems with masses exceeding $15 \mathrm{M}$, down to spectral type BIII. However both samples contain a number of contact systems (such as AO Cas, V729 Cyg, V382 Cyg) or suspected contact (HD 19820, 14 Cen), as well as those systeins recognized as Algol variable (such as SZ Cam, LY Aur, I Sco, see Giuricin, Mardirossian and Mezzetti, 1983). Concentrating on Garmany, Conti and Massey's list, 29 systems remain, of which 18 are double-lined. The overall characteristics of this sample are given in Table 1. For the average value of Msin $i$ we took primary and secundary stars separately.

Remark that there is no double-lined system exceeding a period of 33 days and only two single-lined systems exceeding this value.

\section{TABLE 1}

General characteristics of a sample of unevolved 0-type binaries, taken from Garmany, Conti and Massey (1980)

\begin{tabular}{|c|c|c|c|c|c|}
\hline Parameter & Average & Min. & System. & $\overline{M a x}$ & System. \\
\hline Period (d) & 15.9 & 2.1 & DHCep & 154.9 & $\mathrm{CMa}$ \\
\hline Perigd (d) & 11.0 & 2.1 & DHCep & 34.5 & $\mathrm{HD} 166734$ \\
\hline$q^{(1)}$ & 0.64 & 0.35 & HD206267 & 1.00 & HD47129 \\
\hline$M_{S} \sin 3^{i}\left(M_{0}\right)$ & 17.6 & 0.6 & HD48099 & 58 & HD47129 \\
\hline$M_{s}^{s} \sin i\left(M_{0}^{0}\right)$ & 13.3 & 0.4 & HD48099 & 64 & HD47129 \\
\hline
\end{tabular}

(1) on1y SB2 systems,

b) Evolved binaries with a WR-component.

These systems are dominated by mass loss, an evolved mass ratio and the appearence of nuclear processed products in the spectrum. For the general characteristics we restrict ourselves to the 17 double-lined spectroscopic systems, given by Hidayat et Admiranto and van der Hucht (1984), though the number of single-lined systems is quite as large (5 with large mass function, 13 with small mass function). However, our knowledge on the physical dimensions of the latter systems is quite poor. of the former group, the general appearence is given in table 2.

The average period is 85 days, but 10 out of 17 systems have periods smaller than 10 days. If we look to the WN and WC systems separately, we find that WN systems have smaller periods than WC systems, 
centered around 6 days and 27 days respectively. This is consistent with the idea of evolution from the WN to the WC type (Doom, De Greve and de Loore, 1985), as a result of strong stellar wind loss, causing the period to increase.

\section{TABLE 2}

General characteristics of a sample of double-lined WR +0 binaries, taken from Hidayat, Admiranto and van der hucht (1984).

\begin{tabular}{|c|c|c|c|c|c|}
\hline Parameter & Average & Min. & System & Max. & System \\
\hline Period (d) & 85 & 2.1 & $\begin{array}{c}\text { WR 151 } \\
(\text { WN } 5+08)\end{array}$ & 1085 & $\begin{array}{c}\text { WR } 140 \\
(\text { WC } 7+04)\end{array}$ \\
\hline$M_{W R} \sin 3_{i}$ & $\begin{array}{r}2.4 \\
10.6\end{array}$ & 1.2 & WR 47 & 6.25 & WR9 \\
\hline
\end{tabular}

Remark that none of the systems has a mass ratio smaller than 1 , although in earlier publications some appear as such (CQ Cep : $q=0.84$; Massey, 1982 - HD 92740 : $\mathrm{q}=0.37$; Niemela, 1979, Conti, Niemela and Walborn, 1979, de Jager, 1980) and that they have been theoretically predicted (Doom and De Greve, 1981).

c) Evolved binaries with a compact component, the massive $X$-ray binaries.

Only 8 systems can be considered as 'well studied'. Their general characteristics are given in Table 3. For the period X Per was excluded, because its estimated period of 580 days is still uncertain (de Loore, Altamore and Baratta, 1979). The masses are selected from a search through the literature and should be considered with caution. For the extrema various values are found in the literature:

- For the value of $12.5 \mathrm{i}$ for SMC X-1, found by Conti (1978), alternatives are given by Primini, Rappaport and Joss (1977, 15 to $24 M_{0}$ ) and Rappaport, Joss, and Stothers (1980, $18 \mathrm{M}_{\mathrm{o}}$ ).

\section{TABLE 3}

General characteristics of 8 massive X-ray binaries

\begin{tabular}{|c|c|c|c|c|c|}
\hline Parameter & Average & Min. & System & Max. & System \\
\hline $\begin{array}{l}\text { Period }(d)(1) \\
\text { Mass }\left(M_{f}\right) \\
\log L_{x} / L_{0}\end{array}$ & $\begin{array}{l}4.16 \\
24.5 \\
3.1\end{array}$ & $\begin{array}{r}1.5 \\
12.5 \\
0.4\end{array}$ & $\begin{array}{l}\text { LMC } X-4 \\
\text { SMC } X-1 \\
X \text { Per }\end{array}$ & $\begin{array}{l}8.96 \\
30 \\
5.2\end{array}$ & $\begin{array}{l}\text { Vela } x-1 \\
\text { Cen } X 3 \\
\text { SMC } x-1\end{array}$ \\
\hline
\end{tabular}

(1) X Per excluded. 
From all these systems the nost essential information derived from the binarity is the mass. Its importance is reflected by the fact that due to quantitative derivations the existence of neutron stars was proven (example Vela X-1, Van Paradijs et al. 1977) as well as strong evidence for the existence of a black hole in the system Cyg $X-1$ (Avni and Bahcall, 1975).

\subsection{Related objects.}

Apart from the three groups described above, a number of objects have to be mentioned here, because they may be related to massive binaries and possibly have a binary nature or a binary origin.

a) Runaway stars : divided in OB and WR runaways. Their curious characteristics (high latitude and large velocity) may be explained as the aftermath of the explosion of the now invisible (or disappeared) component somewhere in the past (Blaauw,1960; van den Heuve1, 1968), or they may be swung away the parent association by narrow passage of another star.

This idea has recently by developed by Gies (1985) analysing new extensive radial velocity measurements.

b) Double pulsars, such as PSR $1913+16$ (Taylor and Hulse, 1974), of which the origin is found in a massive system (de Loore, de Loore, de Greve and De Cuyper, 1975; van den Heuvel, 1983).

c) Strange complex objects such as SS433 (Ruffini, 1982).

\subsection{Fraction of binaries among luminous stars.}

A second reason to discuss about massive binaries is that they represent a considerable fraction of the luminous stars. Conti et al. (1983) find a percentage of $36 \%$ of binaries among 424 0-type stars. According to Massey (1982) the WR-binaries constitute one quarter of all WR stars. Hidayat, Admiranto and van den Hucht (1984) estimate this fraction at $35 \%$, for stars in the solar neighbourhood (nearer than $2.5 \mathrm{kpc}$ ). Using evolutionary tracks with extended core models Doom, De Greve and de Loore, (1985) theoretically predict a fraction of 40 to $45 \%$, but if the lower boundary of the initial mass of the WR-star is changed into to $20 \mathrm{M}_{0}$ (in the first estinate $15 \mathrm{M}$ is used as lower boundary) the fraction amounts to $30 \%$. To this has to be added that nost of the WR-stars in the Small Magellanic Cloud, although few in number, seem to be member of a binary system (Azzopardi and Breysacher,1979; see Massey, this volume).

The binary frequency of WR-stars depends on the efficiency of the different formation mechanisins of these stars: stellar wind mass loss or mass transfer (RLOF). More and more evidence is found that this efficiency itself depends on the metallicity of the interstellar environment. From the analysis of the binary frequency of WR stars in external galaxies by Lequeux(1983), shown in table 4, we conclude that the binary fraction is larger in galaxies (or regions in our own galaxy) 
with low Z-value. This implies that in a low $\mathrm{Z}$ region, mass transfer is the dominant formation mechanism, the stellar wind mass loss rate probably being too small to modify the atmospheric abundances. This corresponds with the results of Maeder (1980), who found that the ratio $N(W R) / N(M), M$ refering to M-type supergiants, was very sensitive to metallicity. The correlation between $M$ adn $Z$ was also discussed by Prevot et al. (1980) and Abbott (1982).

\section{TABLE 4}

Binary frequency of WR-stars in external galaxies. (Lequeux, 1983)

\begin{tabular}{|c|c|c|c|c|}
\hline \multirow{2}{*}{ GALAXY } & \multirow{2}{*}{$\begin{array}{l}\bar{\Lambda} \\
\mathrm{I}\end{array}$} & $\bar{N}(W R \quad B I N)$ & \multicolumn{2}{|l|}{$\mathrm{N}(\mathrm{WC})$} \\
\hline & & $N(W R$ TOT) & $\mathrm{N}(\mathrm{TOT})$ & \\
\hline 131 & 0.04 & & 1 & \\
\hline Galaxy $(Z=0.03)$ & 0.03 & 0.15 & 0.52 & \\
\hline Galaxy $(\mathrm{Z}=0.02)$ & 0.02 & 0.24 & 0.44 & \\
\hline$M 33$ & 0.02 & - & $<0.50$ & \\
\hline Galaxy $(Z=0.014)$ & 0.014 & 0.25 & 0.41 & \\
\hline LMC & 0.009 & 0.29 & & 0.20 \\
\hline SMC & 0.003 & 0.63 & 0.12 & \\
\hline
\end{tabular}

3. MODELS FOR MASSIVE BINARIES.

The next question concerning the establishment of generic relations between the different groups is 'what models should we use to describe the evolution?'. The outlook of the system and its evolution depend on the adopted model for the constituent stars, and here a wide variety of possibilities is available (cfr. the review of Maeder, this volume).

a) The very classical massive star, with a convective core determined by equalization of the two dominant temperature gradients responsible for the transport of energy, with constant stellar mass during non-interacting phases. These models were used in close binary evolution up to 1977 .

b) Mass decreasing stars, mimicking stellar wind mass loss with a formalism essentially in agreement with observed mass loss rates. Such models were applied from 1978 on and were considerably improved on the initial mass ratios necessary to obtain the present short period WRsystems (Vanbeveren et al., 1979). 
c) Stars with different inner structure (diffusion, overshooting, extended mixing).

The influence on close binary evolution may best be shown by the difference in initial-to-final-mass relations, connected with these models (initial = ZAMS, final = after RLOF). Let's put it the other way round. Consider a WR star observed as member of a binary system, with an estimated mass of $10 \mathrm{M}$. What is its initial mass? The different possibilities are depicted ${ }^{\circ}$ in Figure 1.

For simplicity we have assumed that the system has evolved through a case $B$ of RLOF, hence mass transfer occurring after the moment the most massive star has left the main sequence (Kippenhahn and Welgert, 1966). We considered two cases:

1 - the star has just entered the WR-phase after a phase of mass transfer : $M_{\text {R }}=M_{f}$ (dotted lines).

2 - WRe stâr has lost about $1 / 3$ of 1 ts mass during part of its WRphase : $M_{W R}=10 M_{0}, M_{f}=13 M_{0}$ (full lines).

The results with the different models $a, b, c$, described at the beginning of this section are labeled $a, b, c$ in the figure.

\section{Figure 1.}

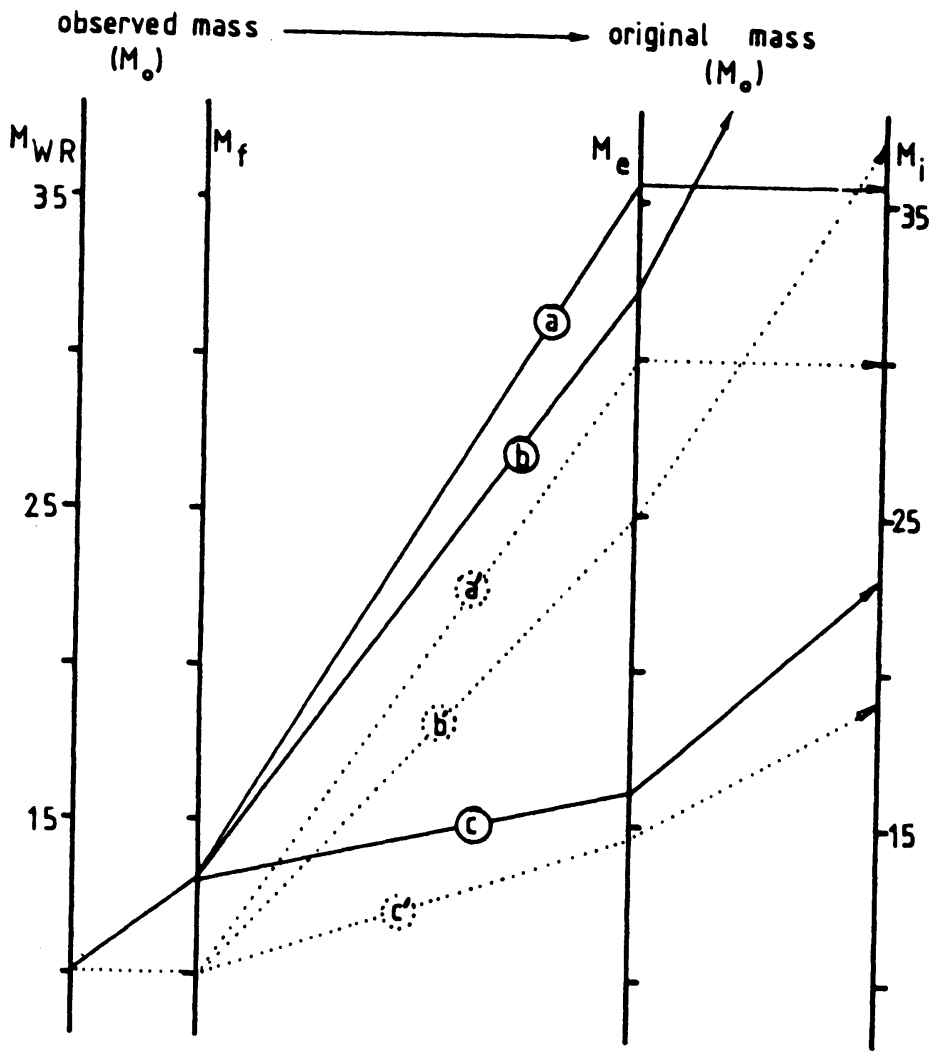


Summarizing we find that a presently observed $W R$ star of $10 \mathrm{M}_{\mathrm{O}}$ may have originated from an initial mass of 19 to $48 \mathrm{M}$, depending on the stellar model used in the computation. We emphasize that with model b simple stellar wind mass loss will not transform the massive component into a WR-star as proposed by Conti (1976).

In the following we will use the models $b$ and $c$ to explore the evolution of massive systems.

\section{CLOSE BINARY EVOLUTION.}

The earlier importance of computations on massive close binary evolution is undoubtedly found in the confirmation of the hypothesis of van den Heuvel and Heise (1972) on the origin of massive X-ray binaries. Schematically the scenario occurs as follows (de Loore, De Greve and De Cuyper 1975).

\begin{tabular}{lllll}
\hline Proces & $:$ & RLOF SN & & \\
SYSTEM & $:$ & $0+0$ & WR binary & $\mathrm{X}$-ray binay \\
\hline
\end{tabular}

The intial masses considered in the computations carried out in the mid-seventies, range from 15 to $30 \mathrm{M}$. As a consequence the derived masses of the WR companions were estimated in the range 5 to $10 \mathrm{M}_{0}$, values that were not contradicted by observations at that time!

Examining this scenario in somewhat more detail we can ask 'Is that scenario true for all o-type systems?', $i . e$. will each 0 -type system be transformed into a WR-system and later on into an X-ray binary?

Let us consider a massive binary system which is evolving from the ZAliS up to an advanced stage. As system characteristics we adopt the mass of the initially more massive star $M_{1}$, the mass ratio $q=M_{2} / M_{1}$, and the period variation $P / P_{i}$. The mass range considered for $M_{1}$ is 15 to $100 M_{0}$. To simplify the picture we adopt $q_{1}=0.7$ for the whole mass range. We wili discuss the different bifurcations in the evolution resulting from the use of two different models and assumptions on the mass transfer.

The following stages are considered :

A : ZAMS

$B$ : End of core hydrogen burning of the most massive star

$C$ : End of RLOF and/or onset of WR stage

$D$ : End of WR stage (=end core helium burning)

$D^{\prime}:$ End $\mathrm{SN}$-explosion $\left(\mathrm{M}_{1}=2 \mathrm{M}_{0}, \mathrm{P}=\mathrm{P} \times 1.5\right)$

$\mathrm{E}$ : End of core hydrogen burning of the companion star.

From computations with models $\mathbf{b}$ and $\mathbf{c}$ respectively, one may infer that :

1- the evolution leads to an overall period increase with almost one order of magnitude

2- with extended cores the mass of the companion star remains nearly unchanged (as the influence of mass transfer, if present, is small) 
or is slowly decreasing to a value of $65 \%$ of the initial value as a result of stellar wind.

3- unless very severe angular momentum loss is considered, it is not possible to obtain advanced systems with small periods $(P<10$ d) starting from periods $P_{i}>10 \mathrm{~d}$.

It seems therefore unlikely that 0-type systems with large masses and periods evolve into $X$-ray binaries, at least what is concerned the samples of well-known binaries.

Now we may try to answer the question: what are the general characteristics of massive close binary systems at the various stages of their theoretical evolution? Figure 2 shows the results for model $b$, with a classical convective core and stellar wind, and model c, with an extended core and stellar wind, with the following constraints:

1- the stellar wind mass loss (for $O$ and WR stars) is assumed to be spherical by symmetric. The period increase is

$\begin{array}{ll}\text { computed from } & P \\ & -\end{array}=\left(-\frac{M_{10}+M_{20}}{M_{1}+M_{2}}\right)^{2} \quad$ (iladjidemetriou, 1967)

with subscript o referring to starting values.

2- when mass transfer is involved, case $B$ is assumed (after core hydrogen burning of the most massive star). In the non-conservative case the period variation is calculated using the formalism of Vanbeveren et al. (1979), adopting a value of alpha $=2$ (corresponding to moderate angular momentum loss from the system, typically $50 \%$ ).

3-as mass loss rate during the WR stage we adopted $310^{-5} \mathrm{M}_{\mathrm{orr}} \mathrm{yr}^{-1}$.

The mass decrease of the Wolf-Rayet is truncated at $4 \mathrm{M}$. The 1ifetime of the WR star is taken equal to the core helium burning timescale.

4- the remnant mass after a Sil-explosion is $2 \mathrm{M}$. A period increase of 1.5 times the pre-SN period is assumed after resynchronisation of the orbit (Sutantyo,1974; De Greve, de Loore and van Desse1, 1978).

A very remarkable result appears in the diagrams of the models with extended cores. First we note that the interval $15-35 \mathrm{M}$ contains some 5 times more stars than the interval $35-100 \%$. Assuming that the fraction of binaries is constant over the mass range $15-100 \mathrm{H}_{0}$ this implies the same difference in number of binary systems. Moreover we know that in the case of a spherical symmetric SN explosion the system remains bound if the removed mass is less than half the total mass of the system. Neglecting impact and influences of asymmetric explosions we find that for $\mathrm{q}_{i}=0.7$ or smaller the removed mass during the Si explosion is roughly half the mass of the system (within $30 \%$ ) for $M_{i}>40 \mathrm{M}_{0}$.

\section{Figure 2 .}

Characteristics of massive close binary evolution with $15<\mathrm{N}_{i}$ / $M_{0}<100 \mathrm{ir}$ and $\mathrm{q}_{i}=0.7$.

Left : classical models with Schwarzschild convective cores. Right :models with extended cores (Roxburgh criterion). 
Dotted area: main sequence evolution of the most massive star, losing stellar wind.

Vertically hatched area: mass transfer (conservative case $B$ ), the dashed curves in the mass ratio diagrain (left) show the final positions in case 50\% (upper curve) or all (lower curve) the transferred mass is lost from the system.

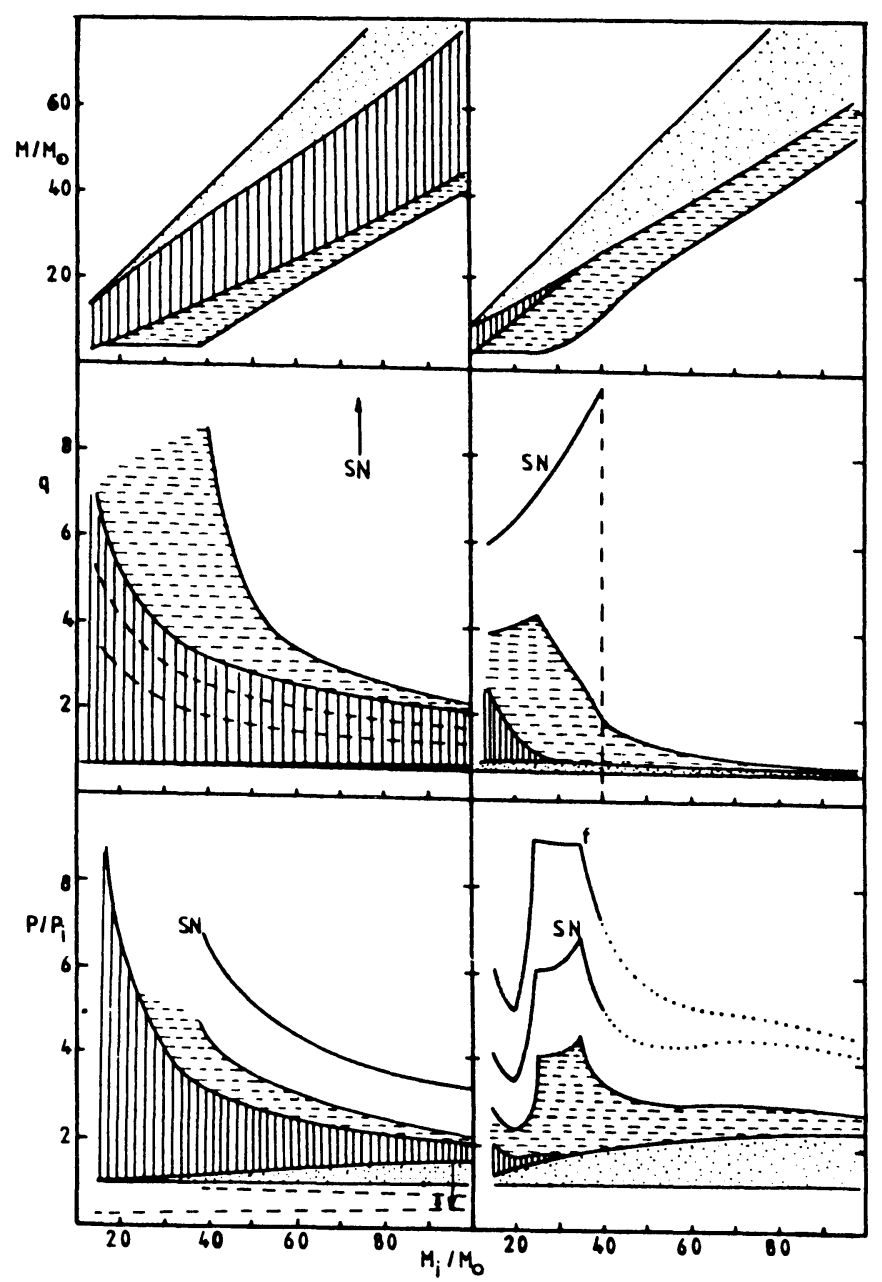

The dashed curves in the period diagram represent the end of mass transfer when all the expelled mass leaves the system (lower curve) and the end of the ensuing WR phase (upper curve).

Horizontally dashed aréa : Wolf-Rayet phase of the originally more massive star ( $M=310^{-5} \mathrm{M}_{0} \mathrm{yr}^{-1}$, with timescale equal to the core helium burning phase). A lower boundary cut-off of $4 \mathrm{M}$ is adopted.

The curve labeled SN is the period increase after the SN explosion and synchronisation of the orbit ( $x b \times 1.5)$; the dotted parts denote the low probability; $f$ is the final period increase at the end of the main sequence of the companion star. 
The vertical dashed line (q-diagrain, right) also denotes a probable boundary for bound systems.

Combining both effects we obtain a large disruption probability for $M>40 M$. Hence in that mass range the appearence of massive $X$-ray binaries is ${ }^{\circ}$ very unlikely. This conclusion should be added to the one obtained from period and secundary mass considerations.

The classical models predict large mass ratios during the WR-stage (overall $q>2$, and for $M_{i}<40 M_{0}$ : $q>3$ ) unless severe mass loss from the system is assumed. ${ }^{1}$ A mass ratio smaller than 1 is not encountered. For models with extended cores the mass ratio remains small for $M_{i}>40 M_{0}$ ( $q 1$, with $q<1$ for $M_{i}>70 M_{0}$ ). For smaller masses the mass ratio is 4.5 at maximum (given the initial value $\mathrm{q}_{j}=0.7$ ).

The classical models predict $\dot{x}$-ray binaries with small periods only in the case of extensive angular momentum loss from the system. For extended core models with initial mass $M_{i}>40 M_{0}$ (in case the system should remain bound after the $\mathrm{SN}$ explosion) a massive $\mathrm{X}$-ray binary results with a period 4 to 6 times the initial period.

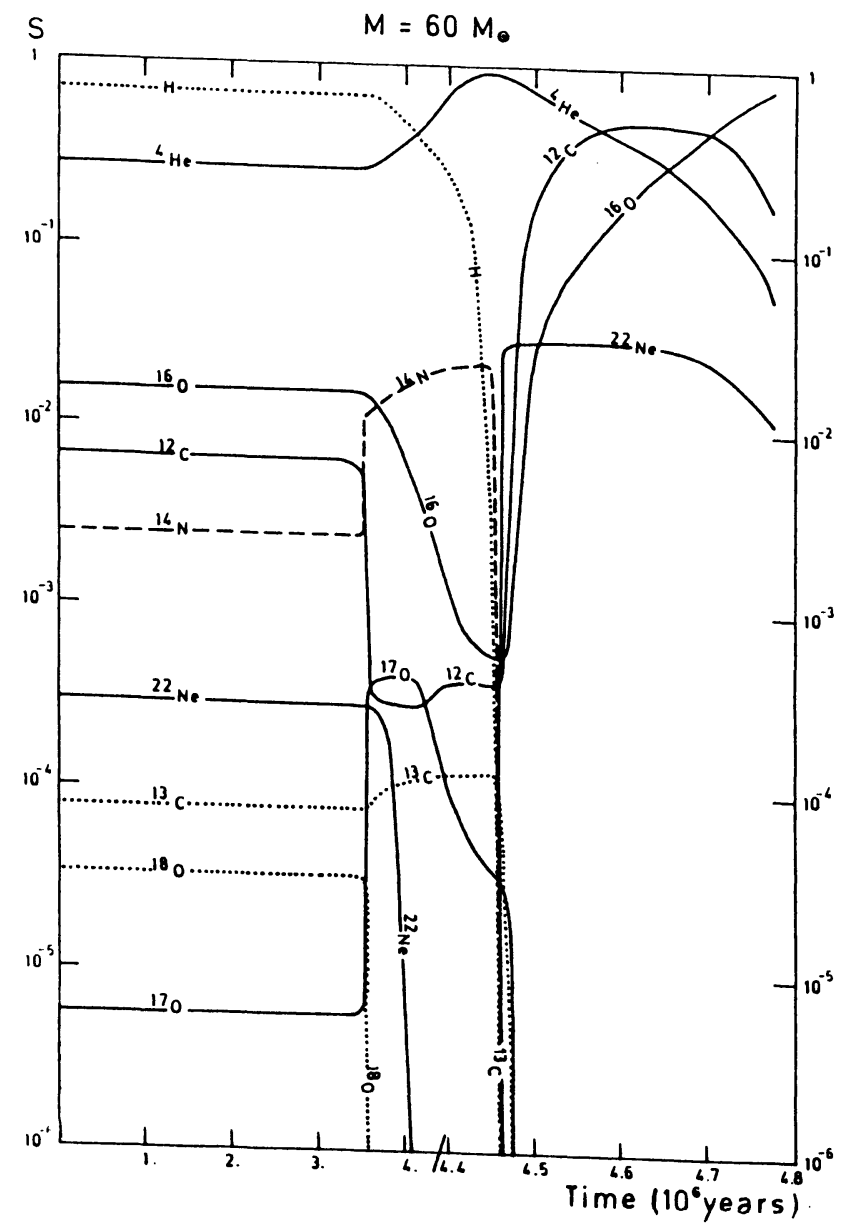


Summarizing we believe that all 0-type systems evolve into WR+0 (or very rarely B) systems, the more massive ones $\left(M_{1 i}>40 M_{0}\right)$ through direct transformation by stellar wind mass loss ( 0 of WR), the less massive ones $\left(M<40 M_{0}\right)$ through mass transfer. Only systems of the latter group will further evolve into massive $X$-ray binraies.

In view of the short periods of the $\mathrm{X}$-ray binaries ( 1.5 to 9 days) they are likely the result of a previous mass transfer process. As such the chemical composition of the surface of both components offers an opportunity to study the nucleosynthesis in the stellar interior as well and to test models of close binary evolution.

Exceptions to this general scheme may result from very massive systems with small periods (implying case A of mass transfer; Doom, 1984) or systems with rather extreme mass ratios.

5. Recommended future work in the field.

From the observational side 2 large regions of interest need extensive further investigation

- an extended survey of unevolved massive systems with reliable dimensions and with correlation to the single stars in the same region would deliver the necessary data to predict statistical properties of advanced stages of those systems.

- detailed abundance analysis of members of the different groups of massive binaries (Unevolved, WR, X-ray, ...) will lead to improved models of mass transfer, surface mixing and mass loss from the system.

Related to theoretical evolution it would be interesting

1) To carry out computations of case A of mass transfer in massive systems, taking into account the changes of the two components. The results should be checked against to improved observations in WR- and Xray binaries. (cfr. Nakamura and Nakamura, 1984, Sybesma, 1985, Hellings, 1985). Such computations might explain well observed systems such as V444 Cyg.

2) To investigate in detail the chemical evolution

Central and surface abundances for evolving stars have been calculated by Maeder $(1983, a, b)$ and compared with observations, and more recently evolutionary computations for massive single stars with extended mixing, stellar wind and detailed nucleosynthesis (including the new reaction) have been presented by de Loore et al. (1985) and Prantzos et a1.(1985). Abundance ratios $\mathrm{N} / \mathrm{He}, \mathrm{C} / \mathrm{He}, 0 / \mathrm{He}$ have been derived which agree with earlier computations, except for 0 , where the use of improved reaction rates gives an overabundance of 0 during the final phases. Nitrogen is burned rapidly during the final phases of He-burning, so that no $N$ is left at the surface during the WC stage.

As an example the surface abundances of $\mathrm{C}, \mathrm{N}$ and $\mathrm{O}$ for a $60 \mathrm{M}$ star

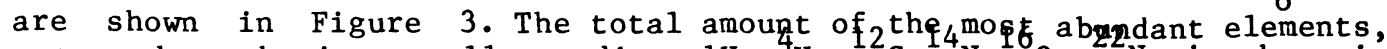
returned to the interstellar medium, $1 \mathrm{H},{ }^{4} \mathrm{He},{ }^{2} \mathrm{C},{ }^{4} \mathrm{~N},{ }^{16} \mathrm{O}^{2},{ }_{\mathrm{Ne}}$ is shown in Table 5 .

Here again the importance of massive binaries for the enrichment 
of the interstellar medium has to be stressed. A massive binary with a not too short period will evolve without mass exchange, and each of the two components will restore a large amount of processed material to the ISM, due to stellar wind mass loss. On the other hand, if mass transfer occurs, part of the matter, expelled by the mass losing component, will be accreted by its companion, and remain stored in its outer layers, until mass loss by stellar wind in this component returns also part of this material to the ISM.

\section{Table 5 .}

$100 \mathrm{M}_{\mathrm{O}}$

\begin{tabular}{|c|c|c|c|c|c|c|c|c|}
\hline$t$ & Phase & Mass & Prot & He4 & $\overline{\mathrm{C} 12}$ & N14 & 016 & $\overline{\mathrm{Ne} 22}$ \\
\hline 2.06 & 0 & 91.8 & 5.7 & 2.2 & 0.049 & 0.026 & 0.12 & 0.002 \\
\hline 4.04 & of & 76 & 11.8 & 11.6 & 0.055 & 0.34 & 0.17 & 0.002 \\
\hline 4.29 & WN & 69 & 12 & 19 & 0.059 & 0.50 & 0.175 & 0.003 \\
\hline 4.39 & WC & 66 & 12 & 19.5 & 0.930 & 0.50 & 2 & 0.06 \\
\hline \multicolumn{9}{|c|}{$50 \mathrm{M}$} \\
\hline 4.62 & 0 & 42 & 5.7 & 2.2 & 0.055 & $0 . \overline{02}$ & $0 . \overline{12}$ & 0.002 \\
\hline 5.57 & of & 37.4 & 8.6 & 4.5 & 0.058 & 0.11 & 0.16 & 0.003 \\
\hline 5.69 & WN & 33.9 & 9.1 & 7.4 & 0.145 & 0.17 & 0.17 & 0.003 \\
\hline 6.08 & WC & 22.3 & 9.1 & 12.4 & 4.16 & 0.17 & 3.73 & 0.117 \\
\hline
\end{tabular}

References.

Abbott, D., 1982, Astrophys. J., 263, 723. Avni, A., Bahcall, J.N., 1975, Astrophys.J. 197, 675. Azzopardi, M., Breysacher, J., 1979, Astron.Astrophys. 75, 120

Blaauw, A., 1961, Bul1.Astron.Inst.Neth. 15, 265. Conti, P.S., 1976, Mem. Soc.Roy.Liege, 6e serie, tome IX., 193.

Conti, P.S., 1978, Astron. Astrophys. 63, 225. Conti, P.S., Niemela, V.S., Walborn, N.R., 1979, Astrophys. J. 228, 206.

Conti, P.S., Garmany, C.D., de Loore, C., Vanbeveren, J., 1983, Astrophys. J. 274, 302 .

De Greve, J.P., de Loore, C., Van Desse1, E.L., 1978, Astrophys.Space Sci. 53, 105 .

de Jager, C., 1980, The brightest stars (Dordrecht, Reide1).

de Loore, C., De Greve, J.P. de Cuyper, J.P., 1975, Astrophys.Space Sci, $36,219$.

de Loore, C., Altamore, A., Baratta, et al. G.B., 1979, Astron. Astrophys. 78, 287 .

de Loore, C., Prantzos, N., Arnould, M., Doom, C., 1985, 5th. Morion, Astrophys. Meeting, Audouze, J. Tran Thanh Van, (eds.).

Doom, C., 1984, Astron. Astrophys. 138, 101.

Doom, C. De Greve, J.P., Astrophys. Space Sci. 80, 369.

Doom, C., De Greve, J.P., de Loore, C., 1986, Astrophys. J. (in press) 
Garmany, C.D., Conti, P.S., Massey, P. 1980, Astrophys. J. 242 .

Gies, 1985, Ph.D.

Giuricin, Mardirossian, Mezztti, 1983, Astrophys.J.Suppl., 52, 35.

Hadjidemetriou, J., 1967 , Adv.Astron.Astrophys. 5, 131 .

Hellings, P., 1985, Ph.D. Thesis, V.U.B. Brussels.

Hidayat, B., Admiranto G., van der Hucht, K.A., 1984, Astrophys. Space Sci 99, 175 .

Kippenhahn, R., Weigert, A., 1967, Z.Astrophys. 65, 251.

Lequeux, J., 1983, in M.C. Lortet and A. Pitault (eds.) 'Wolf-Rayet Stars : Progenitors of Supernovae?, p. 19.

Maeder, A., 1980, Astron.Astrophys. 92, 101.

Maeder, A., 1983, Astron. Astrophys. 120, 113.

Maeder, A., 1983, Astron. Astrophys. 120, 135.

Massey, P., 1982, in C. de Loore and A. Willis

(eds.), 'Wolf-Rayet Stars, Observations, Physics and Evolution', IAU Symp. 99 (Dordrecht, Reide1) p. 251.

Nakamura, M., Nakaruma, Y., 1982, Astrophys. Space Sci. 104, 163.

Niemela, V.S., 1979, in P.S. Conti and C. de Loore (eds.) Mass Loss and Evolution of 0-type Stars', IAU Symp. 83, (Dordrecht, Reidel), p. 291.

Prantzos, N., de Loore, C., Doom, C., Arnould, M., 1985, 5th Moriond Astrophys. Meeting, J. Audouze, J.Tran Thanh Van eds.

Prevot, L., Laurent, C., Paul, J., Vida1-Madjar, A., Audouze, J., Ferlet, R., Lequeux, J., Maucherat-Joubert, M., Prevot-Burnichon, M.L., Rocca Volmerange, B., High ionized species in the spectra of SMC stars, A.A.A. 90, L13, 1980 .

Primini F., Rappaport, S., Joss, P.C., 1977, Astrophys. J. 217, 543.

Rappaport, S.A., Joss, P.C., Stothers, R., 1980, Astrophys. J. 235, 570.

Ruffini, R., 1982, in Z. Kopal and J. Rahe (eds. 'Binary and Multiple Stars as Tracersof Stellar Evolution (Dordrecht: Reide1), p. 373.

Sutantyo, W., 1974, Astron. Astrophys 35, 251.

Sybesma, C.: 1985, Astron. Astrophys. 142, 171.

Taylor, J.H., Hulse, R.A., 1974, IAU Circ. No 2704.

Vanbeveren, D., De Greve, J.P., van Desse1, E.L., de Loore, C., 1979, Astron.Astrophys. 73, 19 .

van den Heuvel, E.P.J., 1968, Bull.Astron.Inst.Neth. 19, 326.

van den Heuvel, E.P.J., 1983, in W.H.G. Lewin and E.P.J. van den Heuvel (eds.) 'Accretion Driven X-ray Sources' (Cambridge:University Press), p. 303 .

van den Heuvel, E.P.J., Heise, J., 1972, Nature Phys. Sci. 239, 67.

Van Paradijs, J., Zuiderwijk, E.J., Takens, R.J., Hammerschlag-Hensberge, G., van den heuvel, E.P.J., de Loore, C., 1977, Astron. Astrophys. Suppl. $30,195$. 
Discussion : C. DE LOORE

\section{DE JAGER}

You show that $O B$ binaries with initial masses exceeding 35 to 40 $M$ can evolve, via the WR stage into 0 stars with a compact core, I assume without a disruption phase in between. But this does not mean that such stars could not eventually, evolve into a supernova, is it not?

\section{SREENIVASAN :}

I believe that you have shown in your presentation, using the logic employed in arriving at your conclusions, that for stars more massive then 40-50 $\mathrm{M}$ at Zero age do become WR stars, binarity is not necessary and for those $e^{0}$ that are less massive RLOF is required to make WR stars. This does not conflict with the conclusions from single star evolution that the minimum mass for stars to become WR stars is around $40 \mathrm{H}_{0}$

Could we then agree that binarity is not a necessary prerequisite for WR star formation?

\section{DE LOORE:}

You are absolutely right. Wolf-Rayet stars can be produced by various channels, either by stellar wind mass loss, where the mass loss rate, hence the peeling off of the outer layers leading to heliumenhanced atinospheres, is dependent on the stellar mass, or in binaries, where similar changes in the atmosphere occur, for the most massive ones again by stellar wind, for less massive ones, and depending on their orbital periods, according to a different mechanism, by Roche lobe overflow. In this case the mass transfer rate is independent on the stellar mass, but rather on the nass ratio. So for the production of single Wolf-Rayet stars one needs stars of very high mass, for binaries, the stellar mass can be lower. But in any case, single Wolf-Rayet stars, as well as Wolf-Rayet binaries can be produced by mass loss in stars. Hence it is exact that binarity is not a necessary condition for the production of Wolf-Kayet binaries. 